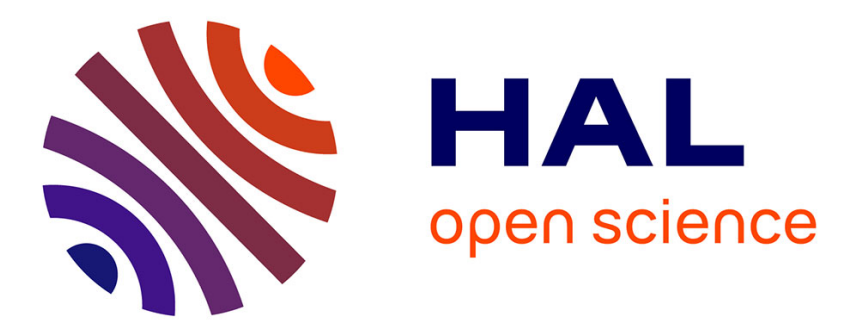

\title{
Electromagnetic Simulations of Radar Backscatter from Tropical Forests: Effects of bio/geo-physical parameters
}

P. Borderies, A. Hamadi, Ludovic Villard, C. Albinet, Thierry Koleck

\section{To cite this version:}

P. Borderies, A. Hamadi, Ludovic Villard, C. Albinet, Thierry Koleck. Electromagnetic Simulations of Radar Backscatter from Tropical Forests: Effects of bio/geo-physical parameters. IEEE International Conference on Numerical Electromagnetic Modelling and Optimization (NEMO 2014), May 2014, PAVIE, Italy. hal-01064971

\section{HAL Id: hal-01064971 \\ https://hal-onera.archives-ouvertes.fr/hal-01064971}

Submitted on 17 Sep 2014

HAL is a multi-disciplinary open access archive for the deposit and dissemination of scientific research documents, whether they are published or not. The documents may come from teaching and research institutions in France or abroad, or from public or private research centers.
L'archive ouverte pluridisciplinaire HAL, est destinée au dépôt et à la diffusion de documents scientifiques de niveau recherche, publiés ou non, émanant des établissements d'enseignement et de recherche français ou étrangers, des laboratoires publics ou privés. 


\section{Electromagnetic Simulations of Radar Backscatter from Tropical Forests: Effects of bio/geo-physical parameters}

\author{
Pierre Borderies $^{1}$, Alia Hamadi ${ }^{1,2}$ \\ ONERA \\ Office National d'Etudes et Recherches Aérospatiales \\ Centre de Toulouse, 31055 Toulouse (France) \\ E-mail : pierre.borderies@onera.fr
}

\author{
L. Villard ${ }^{2}$, C. Albinet ${ }^{1,2}$, T. Koleck ${ }^{2}$ \\ CESBIO \\ (Unité Mixte de Recherche CNRS-UPS-CNES-IRD) \\ Toulouse 31401, FRANCE \\ Email: ludovic.villard@cesbio.cnes.fr
}

\begin{abstract}
This paper deals with the modelling of time series of polarimetric backscattering and temporal coherence of a tropical forest at $\mathbf{P}$ Band. The experimental data show that in the dry season daily cycles of both observables are present and that they are linked to the wind and to the dielectric changes of the canopy. To retrieve these observations, a descriptive model for the forest and an electromagnetic model for the scattering are used. First, canonical studies are carried out about single branches scattering behavior with the goal of highlighting further results. Then, dielectric constant variation and displacements due to the wind are applied to the model and it is shown that they reproduce efficiently the experimental time series.
\end{abstract}

Keywords-electromagnetic modelling; P-Band SAR; Tropical forests; Temporal coherence; biophysical parameters.

\section{INTRODUCTION}

P-band microwaves (wavelength around $70 \mathrm{~cm}$ ) can be used for their capability to penetrate through dense media, such as tropical forests. Being sensitive to the whole vegetation volume, measures derived from P-band SAR (Synthetic Aperture Radar) acquisitions such as the backscattered intensity or the effective canopy height can be correlated to the forest above ground biomass (AGB). Such features have originated the Biomass space borne mission [1] which aims at mapping globally biomass stocks and changes using a SAR operating at $430 \mathrm{MHz}$.

Nonetheless, various geophysical parameters independent from the biomass are liable to impact SAR measurements and thereby to bring perturbations on retrieval algorithms. For instance, the temporal variations linked to vegetation water content (VWC) and wind conditions have been emphasized by the TropiScat Radar experiment[7] (scatterometer on the top of the Guyaflux tower) recently conducted over the Paracou test site (French Guiana). Indeed, the time series of the backscattering coefficient and the temporal Radar coherence acquired during this campaign have exhibited some significant and regular daily changes. It is important to interpret such variations in the context of the Biomass mission. It presents also a broader interest in the retrieval of bio, geophysical properties of tropical forests for other applications and objectives.

For a better understanding of these phenomenons, a sensitivity analysis has been performed from electromagnetic simulations, performed with MIPERS (Multi-static Interferometric and Polarimetric Electromagnetic model for
Remote Sensing)[2][3]. The input data of the model are based on a discrete description of the natural scatterers, incorporating in-situ data and allometric relations. This model affords the polarimetric backscattering coefficient. In addition, it is coherent and so temporal coherences as well as Pol-InSAR coherences can be simulated and enable to quantify the impact of dielectric changes (linked to VWC) or to displacements of the scatterers (due to wind conditions).

In this paper, EM simulations are achieved with the MIPERS model and confronted to the experimental results of the ground based scatterometer TropiScat. Section II recalls the main features of the modelling, first for the individual scatters and later for the global approach. Section III deals with the VWC variations and the wind influence.

\section{ELECTROMAGNETIC MODELLING}

\section{A. Individual Scattering Elements}

At $\mathrm{P}$ band the leaves present a small interaction with the electromagnetic waves so that only branches and trunks are usually represented. The most commonly used approximation to account for them is the homogeneous finite dielectric cylinders. The model used for this purpose here is described in [4]. It can be applied with confidence in P-band since its accuracy has been tested in these conditions of shape and frequency in [5]. Fig. 1 displays the comparis on of the scattering diagram obtains with a numerical method (FDTD), the analytical model and an alternative one. As specular backscattering at normal incidence (with respect to the scattering element) usually drives the global backscattering and specular scattering at the incidence angle drives the double bounce (interaction with ground) contribution, one can check that that the agreement is very good for a typical forest scatter.

Apart from its shape, such scattering diagram is induced by the dielectric constant itself tied to the VWC. Fig. 2 presents the variation of the backscattering coefficient at normal incidence of a dielectric cylinder representing a typical branch: a length of $3 \mathrm{~m}$ and a radius that varies from $0.5 \mathrm{~cm}$ for small, high order branches to a few $\mathrm{cm}$ for primary branches and grows up to trunks dimensions $(25 \mathrm{~cm}$ radius $)$. Note that here $\mathrm{HH}$ and $\mathrm{VV}$ refer the incident wave polarization versus the cylinder positioned vertically. This 
plot is reproduced for various moisture contents from $30 \%$ which corresponds to dry conditions to $80 \%$ for very wet conditions inside the wood, even if the highest values may be somehow exaggerated. These quantities are certainly interrelated since large radius branches are certainly dryer than the extremities which are thin. Actually, distribution of moisture inside a tree is a complex topic since it is variable according to the height of the trunk and in the branches according to the distance to the trunk, and is subject to abrupt diurnal as well as seasonal changes [8]. However, it is interesting to look at the influence of these parameters independently.

For this range of values, in $\mathrm{VV}$, whatever the moisture content, only one resonance peak and subsequent dip appear. The dip becomes weaker as moisture content increases: indeed, in this case, complex permittivity increases both in real and imaginary part, which induces a smaller penetration into the cylinder and also a stronger attenuation inside it. In the resonance zone, the Radar Cross Section is not univocally related to the volume of the scatterer (and hence to biomass): increasing biomass may indeed correspond to lowering RCS. In the Rayleigh zone, before the first resonance peak, there is a very strong increase of RCS with radius. This monotonous growing behaviour exists for all cylinders which radii are less than $4 \mathrm{~cm}$. Beyond the resonance, there is still a growth of RCS with radius (hence with biomass) : the range of radii involved here makes it more interesting for specular direction. This slow growth can be interpreted as the consequence of increasing the effective section of the cylinder in the high frequency regime. Note the very strong differences in $\mathrm{VV}$ and $\mathrm{HH}$ behaviour, both in the Rayleigh and resonance regions, which results in a vertical scatterer roughly saturating at 2$3 \mathrm{~cm}$ whereas a horizontal one saturates at $8-10 \mathrm{~cm}$.

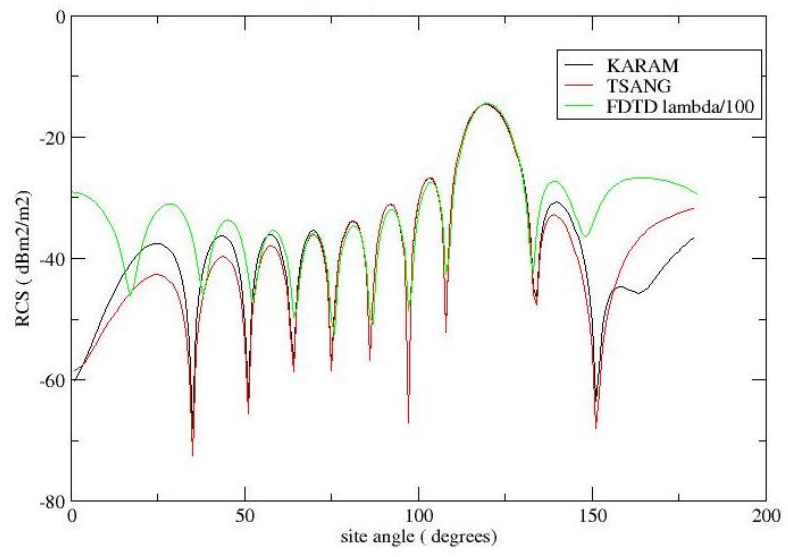

Fig. 1 Scattering diagram in $\mathrm{VV}$ of an homogeneous cylinder of permittivitty $(25 .,-1$.$) , radius 5 \mathrm{~cm}$ and length $1.5 \mathrm{~m}$. Incident plane wave is at $\mathrm{P}$ band and incidence of $30 \mathrm{deg}$. The 3 represented curves correspond to the FDTD and both Karam and Tsang analytical approximations. The diagrams are represented in the incidence plane in the upper hemisphere ( which incorporates the backscatteringdirection). The cylinder is vertical.

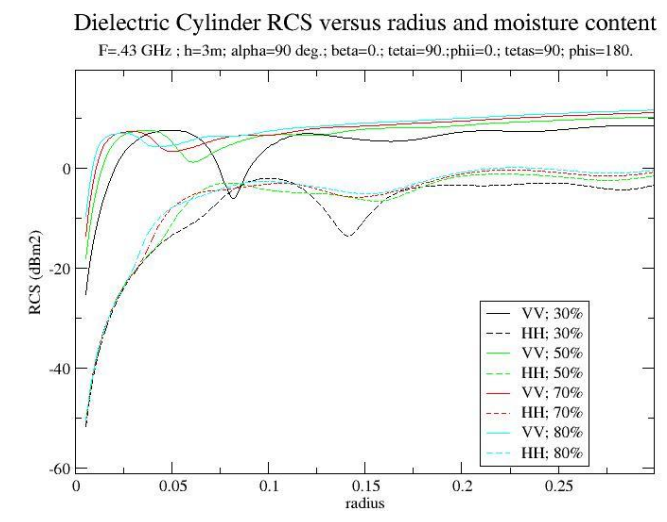

Fig. 2: Variation of the backscattering coefficient of a dielectric cylinder representing a typical branch of length $3 \mathrm{~m}$ and varying radius in $\mathrm{m}$; cylinder axis is vertical and incident illumination is at normal incidence. 4 values of moisture content are considered

\section{B. The Mipers code}

A detailed description can be found in [2] with the adequate references. The model is able to encompass infinite size forests as well as finite ones whatever the radar resolution. To compute the electromagnetic response of the medium, Born extended approximation is used. The contributions of all the scatters present in the scene are coherently summed for the various combinations of all polarizations: this approach is fully polarimetric and phase preserving so it allows full polarimetric, interferometric and POLINSAR simulations.

For each scatter we can then consider several mechanisms:

Volume contribution: As developed in the previous section, each element is envisioned as a discrete scatter of known scattering matrix. The influence of the surrounding scatters is accounted for with Foldy-Lax approximation through the use of an effective propagation constant (attenuation) derived from the forward scattering theorem. Some geometrical computations are performed to account for all intersection points if any between emitter-scatter ray and all vertical and horizontal boundaries of the layers. This process is repeated for the scatter-receiver ray. Then complex transmittivity matrices factor between emitter and scatter and between scatter and receiver are computed taking into account all eventual path lengths in each layer. This scattering component is sensitive to VWC.

Soil contribution: The Integral Equation Method is used. To account for speckle effects and fit with observed data, we spread the backscattering coefficient on the pixel surface with sub-sampling it and attributing to it a random phase, which traduces large scale roughness. This scattering component is sensitive to VWC and to soil moisture, but is scarcely significant.

Volume/soil interaction: For each path considered, similar geometrical computations are implemented and subsequent transmissivity matrices derived. For each mechanism, soil specular reflection is accounted for through modified Fresnel coefficients. This scattering component is sensitive to VWC and to soil moisture. 
The location of the scatters in the volume canopy may be driven by a multi-layer description of the forest or by a tree description which is illustrated by Fig. 3 .

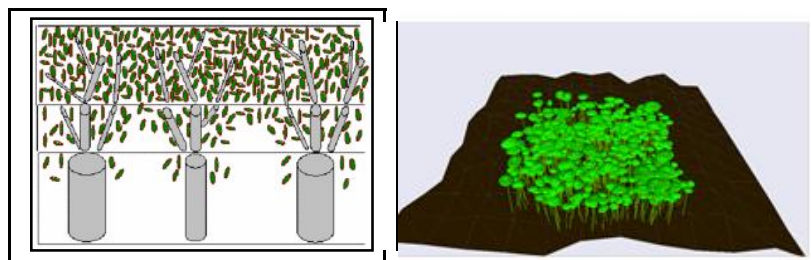

Fig. 3 : Example of tropical forest st and model used by MIPERS to simulate SAR measurements: left, layer description; right: trees set description.

In addition to the above geometrical descriptors, moisture content has to be given for the soil and for each individual scatter, which permits to parametrize the changes in dielectric constants for both soil and vegetation. Note that the construction of the code permits inherently to simulate the effect of displacements induced by wind as was done formerly in [14] for temperate forests.

The output of the code is the complex scattering coefficient, which gives the backscattering coefficient with averaging and the temporal coherence which accounts for the changes of all nature observed in the observed scene between two dates. The value of the coherence between a measurement at a given time $\mathrm{S} 1$ and the reference measurement $\mathrm{S} 2$ at another time is computed with

$$
\begin{gathered}
\gamma_{p q}=\frac{\left|\sum S_{1 p q}(r) \cdot S_{2 p q}(r)^{*}\right|}{\sqrt{\sum\left|S_{1 p q}(r)\right|^{2} \cdot \sum\left|S_{2 p q}(r)\right|^{2}}} \\
\text { with } \quad p=\left|\begin{array}{ll}
H & \text { and } \\
V
\end{array} \quad q=\right| \begin{array}{l}
H \\
V
\end{array}
\end{gathered}
$$

\section{TROPICAL FOREST MODELLING}

\section{A. Observed diurnal cycles}

The description of the experiment can be found in [7] and some results in [10]. Fig. 5 displays some daily cycles of the Radar observables during the dry season. Those results are obtained in the absence of rain, which yields a more simple interpretation. For the temporal coherence, reference is taken at 12 p.m. One can observe a regular decrease during the night up to 6 a.m., and this regularity is retrieved after 6 p.m. up to midnight. At daytime, strong oscillations appear, associated with wind speed increase. In agreement with[13] and our own observations, tropical forests presents a completely different behavior during the night when they look motionless and during the day when their motion is highly apparent. $\mathrm{P}$ band temporal coherence accounts for this typical behavior and then the sources of the decorrelation are filtered: during the night, it is due to dielectric changes and during the day it is due to dielectric changes and to scatters displacements because of wind ( or turbulence).

It is to note that the scene observed by the Tropiscat experiment presents an important slope, of around $10 \%$. Consequently, double bounce contribution is strongly reduced. Then, a minor influence of soil moisture content is expected. This was verified experimentally, since Radar observables were found to be poorly correlated to the interval of time between the measurement and the last rain corresponding to a humidification of soil, but rather to an interval of time suggesting an influence of rain on the VWC of some trees components. For these reasons, the changes in the temporal coherence during the night are considered to be originated by the VWC variation in the branches and trunks. The displacement of the scatters do not affect the intensity, and on the time series of the backscattering coefficient the regularity is not affected by the day/night change: in this case, major source of temporal changes is the dielectric constant evolution due to the evapo-transpiration daily cycles.

\section{B. Impact of dielectric constant changes}

The forest description of the Guyana forest is done according to in-situ measurements and gives the input data of the MIPERS code. For the VWC, measured values over tropical forests are lacking to our best knowledge and thereby the values reported in [8] for temperate forests were chosen. The corresponding complex dielectric constants are displayed on Fig. 4.

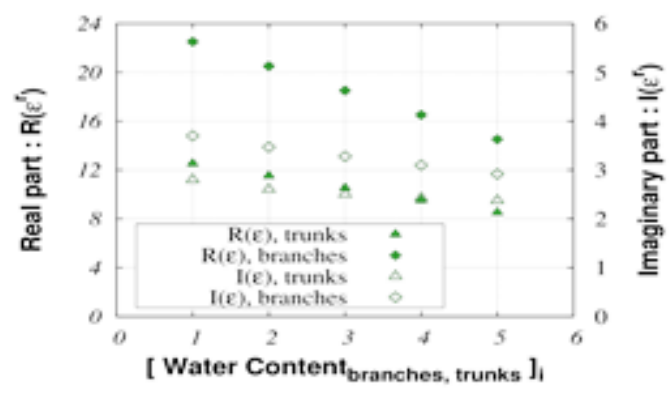

Fig. 4: Complex dielectric values associated to each scenario of VWC

The results of the simulation agree with the changes observed between 12 p.m and 6 a.m. The orders of magnitude are coherent, including the fact that there is a stronger decorrelation in $\mathrm{HH}$ than in the other polarizations.

\section{Impact of Convective Wind}

Based on vectorial modeling of the wind speed field[11][12], the displacements of the scatterers are deduced from the transposition of wind velocity vectors into angular velocities, assuming that only the branches orientation angle is affected. Considering several magnitude of wind speed 

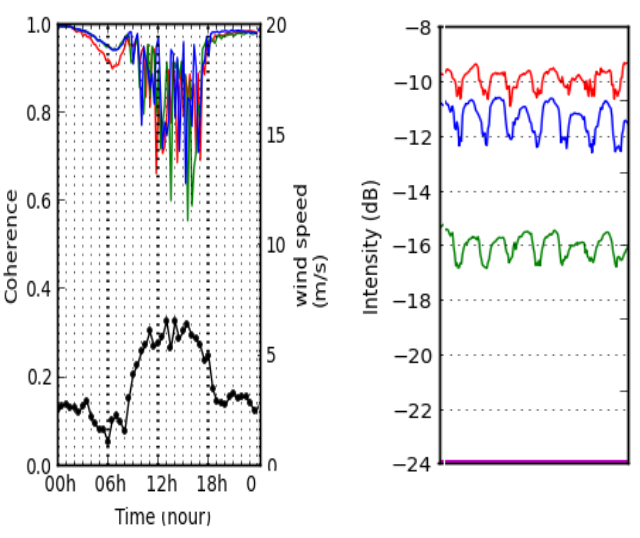

Fig. 5 : Temporal variations in the dry season. Left : coherence derived from $\mathrm{HH}, \mathrm{HV}$ and VV scattering coefficients respectively in red, blue and green during 24 hours ( reference is at 12 p.m.) The bottom curveis the wind speed. Right: backscattering coefficient over a period of 7 days in the dry season.

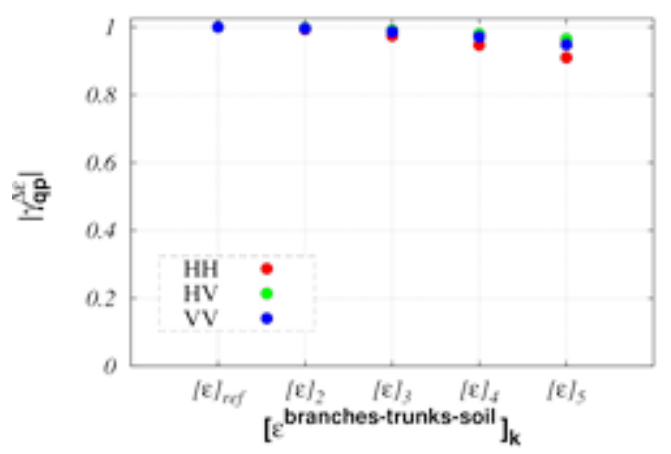

Fig. 6: Decorrelation resulting from the simulated total backscatter due to changing values of permittivity given by Fig. 4

according to those measured during the TropiScat experiment, simulated results are given in Fig. 7 .

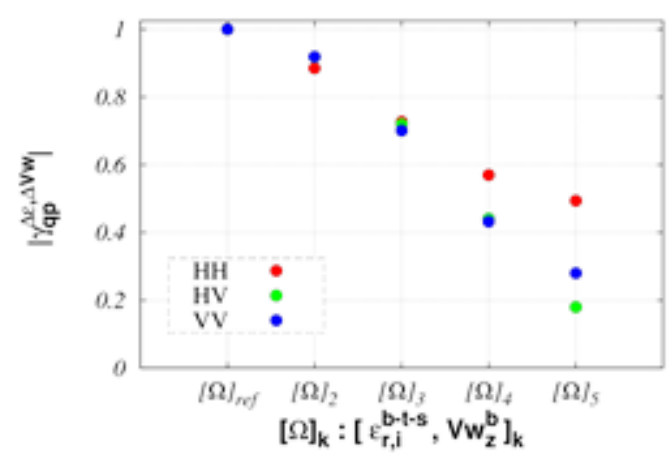

Fig. 7: Decorrelation resulting from the simulated total backscatter due to bigger and bigger wind speed considering the conversion of wind speed into branches orientation changes and spatial displacements.

\section{FUTURE PROSPECTS}

The electromagnetic model has shown its ability to reproduce the time series observed on tropical forests. The benefits which can be withdrawn with its use in system simulators is very broad. Future prospects include the continuation of this analysis and its extens ion to tropical forest over a flat area.

\section{ACKNOWLEDGMENT}

The authors wish to thank CNES for supporting this modelling work in the frame of the TOSCA activities. The authors would like to thank Lilian Blanc and Benoit Burban with 'Ecologie des Forets de Guyane', and Damien Bonal with the Institut National de la Recherche Agronomique, for their welcome and help with the Guyaflux tower.

\section{REFERENCES}

[1] T. Le Toan, S. Quegan, M. Davidson, H. Balzter, P. Paillou, K. Papathanassiou, S. Plummer, F. Rocca, S. Saatchi, H. Shugart, L. Ulander, "The BIOMASSMission : Mapping global forest biomass to better understand the terrestrial carbon cycle," Remote Sensing of Environment, vol. 115, no. 11, pp. 2850-2860, Jun. 2011.

[2] L. Villard, P. Borderies, "Backscattering border effects for forests at CBand," Piers Online, vol 3, no. 5, pp. 731-735, 2007.

[3] L. Villard, P. Borderies, and T. Le Toan, "Topography effects on forest radar scattering, consequences on biomass retrieval," in International Geoscience and Remote Sensing Symposium (IGARSS). IEEE, 2010, vol. 20-24, pp. 60-63.

[4] L. Tsang, J.A. Kong "Scattering of Electromagnetic waves: Advanced Topics", Wiley Interscience, 2001.

[5] ESA contract $\mathrm{n}^{\circ}$ 20449/06/NL/LvH 'Rigorous numeric techniques applied to microwave interaction with natural targets : volume scattering'

[6] F. T. Ulaby, M. A. El-Rayes, "Microwave Dielectric Spectrum of Vegetation - II: Dual-Dispersion Model", IEEE Transactions on Geoscience and Remote Sensing, vol. GE, pp. 550-557, 1987.

[7] C. Albinet, P. Borderies, T. Koleck, F. Rocca, S. Tebaldini, L. Villard, T. Le Toan, A. Hamadi, and D. Ho Tong Minh, "Tropiscat: A ground based polarimetric scatterometer experiment in tropical forests," Selected Topics in Applied Earth Observations and Remote Sensing, IEEE Journal of, vol. 5, no. 3, pp. 1060-1066, june 2012.

[8] K.C. McDonald, R. Zimmermann, and J.S. Kimball, "Diurnal and spatial variation of xylem dielectric constant in norway spruce (picea abies [1.] karst.) as related to microclimate, xylem sap flow, and xylem chemistry,"Geoscience and Remote Sensing, IEEE Transactions on, vol. 40, no. 9, pp. 2063-2082, 2002.

[9] D.M. Gates, "Water relations of forest trees," IEEE Trans. on Geoscience and Remote Sensing, vol. 29, pp. 836- 842, Nov. 1991.

[10] A. Hamadi, C. Albinet, P. Borderies, T. Koleck, L. Villard, D. Ho Tong Minh, and T. Le Toan, "Temporal survey of polarimetricpband scattering of tropical forests," IEEE Transactions on Geoscience and Remote Sensing, vol. 99, pp. 1-9, November 2013.

[11] J. Beaudoin and J. Keyser, "Simulation levels of detail for plant motion," in Siggraph/Eurographics Symposium on Computer Animation. ACM, 2004.

[12] M. Lavalle, M. Simard, and S. Hensley, "A temporal decorrelation model for polarimetric radar interferometers," Geoscience and Remote Sensing, IEEE Transactions on, vol. 50, no. 7, pp. 2880-2888, 2012.

[13] M.S. Assis "Comments on Some radio Wave Propagation Mechanisms in the Amazon region", Piers proceedings, pp. 265-269, Kuala Lumpur, Malaysia, 27-30 Mar. 2012.

[14] C. Ruiz, P. Borderies, I. Chenerie, E. Mougin, C. Proisy “Modelling of electromagnetic waves interaction with forest canopies", CEOS'99, 2629 Octobre 1999, Toulouse. 